\title{
Road marking abrasion defects detection based on video image processing
}

\author{
Zhang Yiheng ${ }^{1, a}$ \\ ${ }^{1}$ China Transport Telecommunications \& Information Center, Beijing, 100011, China \\ a zhangyiheng@cttic.cn
}

Keywords: Road traffic marking damage; Defect detection; Morphology; Line extraction.

\begin{abstract}
Pavement marking occupies an important position in the road traffic safety operation. A large number of domestic and foreign research and practice has proved that the effective use and maintenance of road signs and markings reduce traffic accidents and improve the capacity of great significance. The main content of this paper is the design of mobile video image acquisition device to road damage to the road traffic marking defect detection, video image to carry out research on road marking based on wear and breakage from video images. The process is random image acquisition of pavement markings, respectively the image pre-processing, image binarization, marking angle correction, marking extraction and detection. Finally, developing software on marking the wear and damage degree detection based on Matlab software then have an image processing experiments. The results show that the software can accurately detect the road wear and damage situation.
\end{abstract}

\section{Introduction}

With the rapid development of human science and technology, the car is playing a more and more important role in people's life. However, the development of the automobile has improved people's living standards, but at the same time, it also brings a lot of traffic accidents. Related materials show: only in 2009, China has thousand people died in traffic accidents, accounted for one fifth of the world's total [1]. This makes us alert and aware of the urgency to solve the problem of traffic safety.

According to a large number of domestic and foreign research and practice has proved that the effective use and maintenance of road signs and markings can reduce traffic accidents and increase traffic capacity, save energy, protect the environment of road [2]. Thanks to the rapid development of artificial intelligence computer vision technology, image and video technology has been widely used in intelligent transportation systems and unmanned vehicle systems. Carnegie Mellon University developed a Navlab.X [3] series product system that is based on road flat assumption and using the characteristics of the parallel to the road, such as the lane line, rut, road boundary. University of Parma's PROMETHEUS project has an important part of the GOLD (Generic Obstacle and Lane Detection) [4]. The basic principle of the visual system is using camera as a navigation tool, to analysis the captured image, to detection and extraction of the lanes to avoid obstacles using stereo vision. France Citroen technology center and Pascal university automation and electronic materials science laboratory in France have cooperation in the development Puegoct system [5]. The main principle is using Gauss filter and filter to calculate lane using gradient derivative of the noise left (or right) edge. Real-time and robustness are good. Xin Zhou [6] 
proposed a novel reconstruction lane line algorithm, which is based on the geometric characteristics of lane lines and gray information to construct constraint equations. It only processes the edge of the lane, which can reduce the processing time. Review the literature, the development of intelligent transportation system is based on the recognition of road marking good, but the defects of the pavement markings will affect the recognition of the intelligent transportation system processing. This article is based on the Matlab on the pavement marking for wear, damage detection, develop wear and tear software.

\section{Image preprocessing}

Image preprocessing has three parts: the road image gray, gray stretch, image filtering denoising. Image gray can reduce the amount of color information in the image so that it contains only the brightness information, which simplifies algorithm complexity. Gray level stretching can improve the dynamic range of gray level in image processing. Image filtering is mainly used to eliminate or reduce noise in the image.

\subsection{Image grayscale}

In the RGB color space, red, green, and blue are obtained by a certain mixture. Convert RGB color image to gray image, to quantify the value of brightness. From the experimental experience value, the gray level image can be gained when the ratio of 0.3:0.59:0.11 mixed red, green and blue.

\subsection{The image gray level stretch}

Gray stretch [7] is the most basic gray transformation. The main idea is to improve the dynamic range of gray level image processing. If the image is too dark, it can be used to stretch the gray scale (slope $>1$ ) to improve the image; if the image is bright, it can be used to compress the gray level of the object to improve the image quality by using the gray level stretching function (slope $<1$ ).

Gray stretch results are shown in Fig.1.

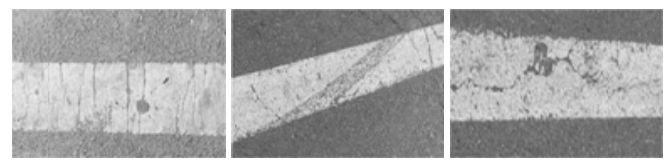

(a)

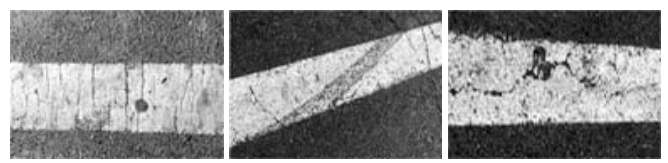

(b)

Fig.1.(a) Before gray stretch.(b)After gray stretch.

\subsection{Image filtering}

The median filter [8] is a template that contains an odd number of points, from left to right, from top to bottom, traversal of each pixel in the image. The pixels in the window are sorted according to the size. Then the median value is obtained. And the median value is used as the gray value of the corresponding pixels in the filtered image. Use median filter for image processing. The filtering effect is shown in Fig.2. 


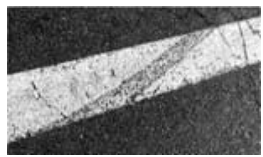

(a)

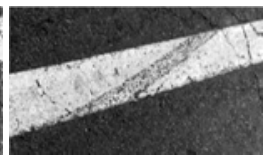

(b)

Fig.2.(a) Before median filter, (b) After median filter.

\section{Binarization and line extraction}

\subsection{Binaryzation algorithm}

Image binaryztiaon is the most important step to select the appropriate segmentation threshold [9]. For grayscale images, one or several gray select the appropriate value of $t(t=0 \sim 255)$ will be separated from the target and the background, the gray value of $t$ is called threshold. If you select a threshold value, it is called the two value of the image. Otsu algorithm [10] is also known as the maximum between class variance methods. The basic idea of Otsu algorithm is to divide the image into two groups (or two kinds) with a certain gray value. The gray value is the best threshold value of the two value of the image when the variance between the two groups is the largest. The results with Otsu algorithm are shown in Fig.3.

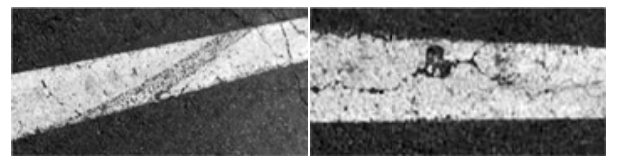

(a)

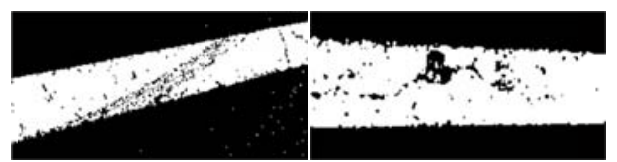

(b)

Fig.3.(a) Before Otsu algorithm processing, (b) After Otsu algorithm processing.

\subsection{Morphological processing}

Mathematical morphology [11] is a new method for image processing and pattern recognition. The basic idea is to measure and extract the corresponding shape image in the structure of a certain form elements to achieve the purpose of image analysis and recognition.

The basic operation [12] of binary morphology includes corrosion, expansion, opening operation and closing operation. The open and close operation is carried out on the basis of corrosion and expansion.

\subsection{Dilation and erosion operation}

Dilation is indicated by symbol $\oplus$, and A dilates B denotes $\mathrm{A} \oplus \mathrm{B}$. The process of dilation as follows: First, mapping the structural elements $\mathrm{B}$ about the origin to get; then, letting have a translation about $x$ to get; finally, calculating the intersection of and binary image(Set A).

Erosion is indicated by symbol $\Theta$, and A erodes $\mathrm{B}$ denotes $\mathrm{A} \oplus \mathrm{B}$. The process of dilation as follows: After Structural elements B having a translation $\mathrm{x}$, it is still in the reference point of the collection A. 


\subsection{Open operation and close operation}

Using structural elements $\mathrm{D}$ to do open operation with the target image $\mathrm{C}$, noting as $\mathrm{C} \mathrm{D}$. The process of dilation as follows: First, eroding $\mathrm{C}$ with $\mathrm{D}$; then, using $\mathrm{D}$ to dilate the results of erosion. Open operation can make the contour of the object being processed becomes more smooth and soft, disconnect the image from the narrow gap, and eliminate the subtle projection.

Using structural elements $\mathrm{D}$ to do close operation with the target image $\mathrm{C}$, noting as $\mathrm{C} \mathrm{D}$. The process of dilation as follows: First, dilating $\mathrm{C}$ with $\mathrm{D}$, then, using $\mathrm{D}$ to erode the results of dilation. Close operation also has the function of smooth contour, but what contrary to open operation is that it usually breaks the narrow and eliminates elongated grooves and tiny holes, and fill cracks in the contour lines as well. Morphological processing result is shown in Fig.4.

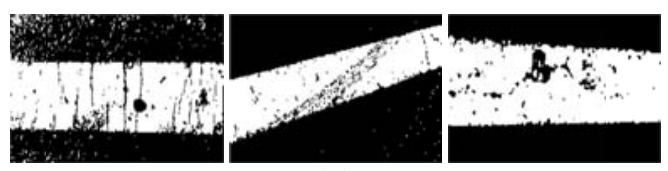

(a)

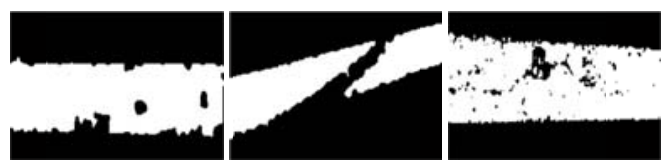

(b)

Fig.4.(a) Before morphological operation, (b) After morphological operation

\subsection{Angle correction}

Scanned image gray value coordinates calculated by the coordinates of the image rotation angle, thus marking angle correction. Corrected image output has two properties crop and loose. The result is shown in Fig.5.

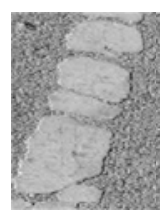

(a)

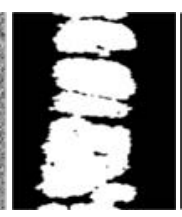

(b)

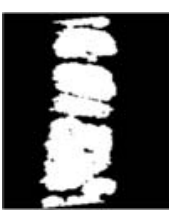

(c)

Fig.5. (a) Gray image, (b) Output of crop attribute, (c) Output of loose attribute The corrected image is shown in Fig.6.

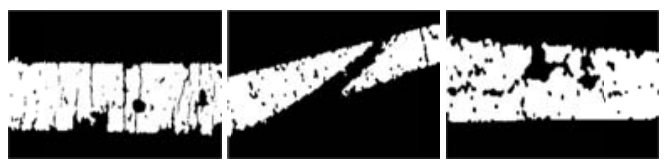

(a)

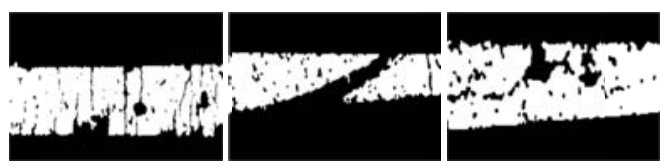

(b)

Fig.6.(a) Before rotation, (b) After rotation. 


\subsection{Line extraction}

After getting the image angle correction, then the image coordinate scanning gray value coordinate marking four vertex value, which will extract the marking.

Marking the extraction results is shown in Fig.7.

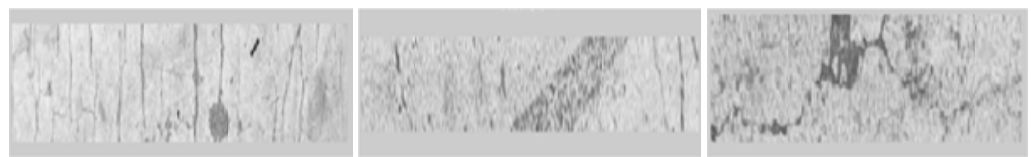

(a)

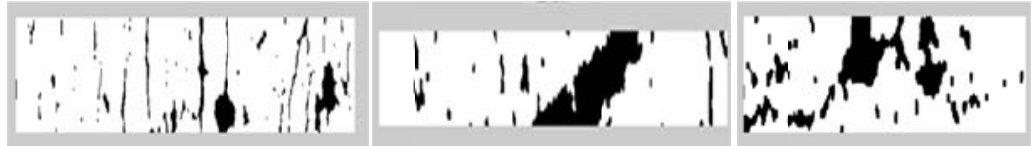

(b)

Fig.7.(a) Gray markings, (b) Binarization marking

\section{Line detection}

\subsection{Line breakage detection}

The method of calculating area can be used to calculate the damage rate by the number of gray values. The gray value of 255 is displayed as white. Gray value is 0 on behalf of black. The results are shown in Fig.8.

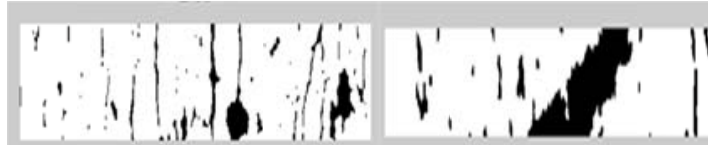

(a) (b)

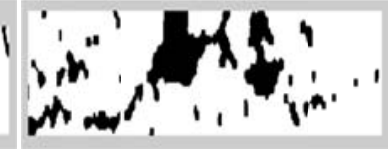

(c)

Fig. 8. (a)Line breakage rate of $8.79 \%$,(b)Line breakage rate of $18.69 \%$,

(c)Line breakage rate of $19.51 \%$

\subsection{Marking wear detection}

After a large number of new line by line, we can get a middle gray value. If the gray of any point in the marking line is greater than intermediate gray value, it is considered to have suffered a certain degree of wear. Otherwise the wear is considered to be light or not wear. The results of the marking test are shown in Fig.9.

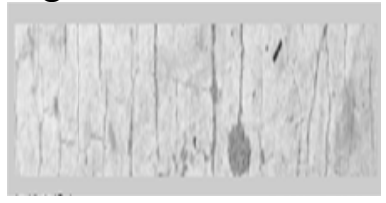

(a)

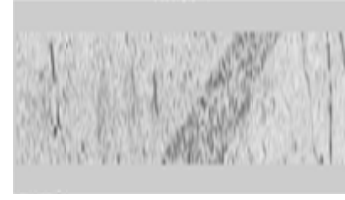

(b)

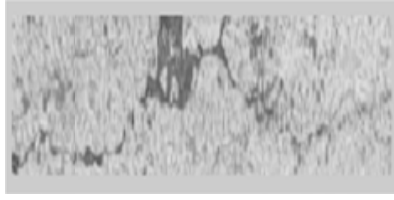

(c)

Fig. 9. (a)The wear rate of marking:8.81\%,(b)The wear rate of marking: $17.73 \%$,

(c)The wear rate of marking:28.55\%

\section{Software development}

Software development is based on Matlab and its main functions include image reading, image preprocessing, image binarization, morphological processing, marking correction and extraction, 
marking wear defect detection.

\section{Conclusions}

The road markings defect detection based on video image processing technology are mainly about image preprocessing, binarization, morphological processing, extraction and marking correction by Matlab process. Next we can get the marking of the wear and damage performance. Based on all those, develop markings wear detection software. Experiments prove that the software can be a very good marking processing and can get marking the wear and breakage accurately.

\section{Acknowledgments}

This work is supported by the science and technology projects of Jiangxi Province Department of transportation: Smart key technology research and application in the highway construction in Jiangxi province (2016C0003).

\section{References}

[1] R. Risack, N. Mohler, W. Enkelmann. A video-based lane keeping assistant. Proceedings of IV 2000 Intelligent Vehicles Symposium, in Dearborn, MI, USA, (2000), p.356-361.

[2] Xiu-min Chu, Xin-ping Yan, Xian-zhen Zhang, Advanced Technologies for Maintenance Management of Highway Sign and Marking, Journal of Transport Information and Safety, in Traffic and computer, vol.23,no.3, (2005), p.104-106.(In Chinese)

[3] Wu Mo, Xiangjing An, Hangen He. On vision-based lane departure detection approach. In IEEE International Conference on Vehicular Electronics and Safety, (2005), p.253-257.

[4] Hold S, Nunn C, Kummert A, et al. Efficient and Robust Extrinsic Camera Calibration Procedure for Lane Departure Warning. In Proceedings of the IEEE Intelligent Vehicles Symposium, (2009), p.382-387.

[5] R.Chapuis. Real-time Vehicle trajectory supervision on the highway. In Int Journal of Robatics Research, vol.14, no.6, (1995), p.531-542.

[6] Xin Zhou, Xi-yue Huang, Xing Li, Lane Keeping and Distance Measurement Based on Monocular Vision, In Journal of Image and Graphics, vol.8,no.5, (2003), p.590-595. (In Chinese)

[7] Yao-fei Tang, The Implementation of Image Gray-Lever Stretch Algorithm Based on FPGA, In Aero Weaponry, no.2, (2010), p.21-23. (In Chinese)

[8] Yan-bing Liu, Research on lane detection method based on directional tunable filter. Master's thesis, Chengdu: University of Electronic Science and Tech, (2009). (In Chinese)

[9] Shao-lin Sun, Zhi-qiang Ma, Wei Tang, Research on Gray-level Image Binarization Algorithms. In Value Engineering, vol.22, no.4, (2010), p.16-19. (In Chinese)

[10] Su-min Yin, Jin-ping Zhu, Zu-shen wang, Research on Image Segmentation Method Based on Top-hat Transformation and Otsu. In Science Technology and Engineering, vol.14, no.7, (2014), p.60-64. (In Chinese)

[11] Juan Wang, Jin-zhi Zhou. Research on morphological image processing based on Matlab. In Modern Society, no.9, (2013), p.4-5. (In Chinese)

[12] Ying Wu. Application of Morphology in Image Processing. In Computer and Modernization, no.5, (2013), p.90-94. (In Chinese) 